\title{
氮掺杂中空碳球氧化物模拟酶性能研究
}

\author{
郑燕宁 ${ }^{1,2}$ ，季军荣 ${ }^{3}$ ，梁雪玲 ${ }^{1}$ ，赖正杰 ${ }^{1}$ ，陈启帆 ${ }^{1}$ ，廖丹葵 ${ }^{1,2}$ \\ (1. 广西大学 化学化工学院, 南宁 530004; 2. 广西大学 广西碳酸钙产业化工程院, 广西钙基材料创新协同中心, 南宁 \\ 530004；3．崇左南方水泥有限公司广西钙基材料创新协同中心，崇左 532201)
}

摘 要: 纳米酶由于其独特、高效、稳定的催化性质而在生化反应中备受关注。本研究以碳酸钙微球为绿色模板剂, 多巴胺为氮源与碳源, 合成了氮掺杂中空碳球 (N-HCSs)。以 3, 3',5,5'-四甲基联苯胺 ( TMB ) 为底物, 采用紫外 分光光度法探究了 N-HCSs 的类氧化物酶的催化活性, 并对其催化机理作了研究。结果表明, N-HCSs 具有氧化物 模拟酶催化活性, KOH 活化后氮掺杂中空碳球的催化活性提高了 3 倍; N-HCSs 氧化物模拟酶催化反应符合 Michaelis-Menten 方程, 活化前后的米氏常数 $K_{\mathrm{m}}$ 分别为 0.109 和 0.083 , 对底物具有良好的亲和能力; N-HCSs 氧 化物模拟酶催化反应中起主要作用的活性氧基团是超氧阴离子 $\left(\mathrm{O}_{2} \bullet^{-}\right)$。本研究为高活性无机非金属类氧化物模拟 酶的设计和制备提供了理论依据。

关 键 词: N-HCSs; 氧化物模拟酶; 反应机理

中图分类号: TQ12 文献标识码: A

\section{Performance of Nitrogen-doped Hollow Carbon Spheres Oxidase Mimic}

\author{
ZHENG Yanning $^{1,2}$, JI Junrong ${ }^{3}$, LIANG Xueling ${ }^{1}$, LAI Zhengiie ${ }^{1}$, CHENG Qifan ${ }^{1}$, LIAO Dankui ${ }^{1,2}$ \\ (1. School of Chemistry and Chemical Engineering, Guangxi University, Nanning 530004, China; 2.Guangxi Cooperative In- \\ novation Centre for Calcium-based Materials (GCICCM), Guangxi Engineering Academy for Calcium Carbonate Industry, \\ Guangxi University, Nanning 530004, China; 3 Guangxi Cooperative Innovation Centre for Calcium-based Materials
}

(GCICCM), Chongzuo South Cement Co. LTD, Chongzuo 532201, China)

\begin{abstract}
Due to high stability and sensitivity, nanozymes have recently attracted much attention in bioreaction. In this work, a facile approach for preparing N-doped hollow carbon sheres (N-HCSs) by using $\mathrm{CaCO}_{3}$ spheres as green template and polydoamine as nitrogen and carbon sources was reported. The morphology and structure of the samples were characterized. Using TMB (3,3,5,5' tetramethylbenzidine) as a substrate, UV sectrohotometry was used to investigate its oxidase-like activity and catalytic mechanism. The results showed that N-HCSs displayed oxidase-like activity. The oxidase-like activity of N-HCSs was increased by three times after activation by $\mathrm{KOH}$. These enzymes conform to the Michaelis-Menten kinetic equation, and the $K_{\mathrm{m}}$ constant before and after activation were 0.1094 and 0.0825 , respectively, indicating good affinity for substrates. The data demonstrated that it is superoxide anion $\left(\mathrm{O}_{2} \bullet^{-}\right)$that plays a major role in catalytic reaction. All these data provides a theoretical basis for the design and preparation of high activity oxidase-mimicking enzymes.
\end{abstract}

Key words: N-doped hollow carbon sheres; oxidase-like enzyme; reaction mechanism

收稿日期：2020-07-04; 收到修改稿日期：2020-08-20

基金项目：国家自然科学基金项目（51372043）; 广西自然科学基金重点项目（2017GXNSFDA198052）;广西大学钲基材料协同 创新研究项目(20190962)

National Natural Science Foundation of China (51372043); Foundation of Guangxi Natural Science key projects (2017GXNSFDA198052); Research Project on Collaborative Innovation of Calcium-Based Materials in Guangxi University (20190962)

作者简介：郑燕宁（1995-)，女，硕士研究生. E-mail: ningningzy@ sina.cn

ZHENG Yanning (1995-), female, Master candidate, E-mail: ningningzy@sina.cn

通讯作者: 廖丹葵, 教授. E-mail: liaodk@gxu.edu.cn

LIAO Dankui, professor. E-mail: liaodk@gxu.edu.cn 
无机纳米材料具有高稳定性、可规模化制备、 成本较低等特点 ${ }^{[1]}$ 。自 2007 年首次报道纳米酶以来, 无机纳米材料不再被认为是生物惰性物质, 已经被 证实具有类氧化物酶、过氧化物酶、过氧化氢酶、 超氧化物歧化酶等催化活性 ${ }^{[2-5]}$ 。纳米酶虽然克服了 天然酶不稳定和昂贵的缺点, 但是部分纳米酶的催 化效率仍然较低, 针对提高纳米酶催化效率的研究 不断涌现 ${ }^{[6]}$ 。大量研究发现, 纳米酶的活性可以通 过控制纳米材料的尺寸、形貌和表面性质进行调控 [7-9]。密度泛函理论（DFT）计算已证实了纳米酶的 催化活性依赖于晶体的尺寸和所暴露的晶面 ${ }^{[10]}$, 而 通过一些元素 ( $N 、 C o 、 Z n$ 等) 掺杂, 可有效提高 碳基纳米酶的催化活性 ${ }^{[11-13]}$ 。这些研究为提高纳米 材料模拟酶的催化活性提供了理论依据。

目前, 纳米酶的研究主要集中在过氧化物模拟 酶方向, 而氧化物模拟酶能够在没有 $\mathrm{H}_{2} \mathrm{O}_{2}$ 的存在下 催化底物 3,3,5,5-四甲基联苯胺 ( TMB ) 发生显色反 应, 应用前景广阔。目前报道的氧化物模拟酶主要 集中在贵金属 $\left(\mathrm{Au}^{[14]} 、 \mathrm{Ag}^{[15]} 、 \mathrm{Pt}^{[16]}\right.$ 和 $\mathrm{Pd}{ }^{[17]}$ 等 $)$ 纳米 材料上, 但是由于其稀缺性而成本较高, 限制了进 一步应用。而过渡金属氧化物(如 $\mathrm{CeO}_{2}{ }^{[18]} 、 \mathrm{MnO}_{2}{ }^{[19]}$ 和 $\mathrm{Co}_{3} \mathrm{O}_{4}{ }^{[20]}$ 等) 也具有类氧化酶活性, 但其在纳米尺 度上易团聚、不稳定, 导致其类氧化物酶催化活性 较低。中空碳球具低密度、大孔容、高比表面积, 在催化、吸附和生物传感等众多领域广泛应用 ${ }^{[21-22] 。}$ 氮元素被掺入到碳纳米材料石墨化晶格时产生晶格 缺陷, 可为催化反应提供更多反应活性位点, 从而 使得氮掺杂碳材料成为一种优良的氧还原反应 （ORR）催化剂[23-24]。氧化物模拟酶催化反应是基

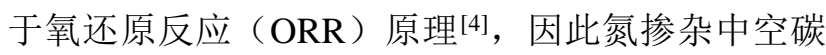
球有望成为高效氧化物模拟酶。硬模板法是制备中 空碳球最简便有效的方法, 二氧化硅 $\left(\mathrm{SiO}_{2}\right)$ 是报 道较多的硬模板剂, 但是其在去除模板时需使用强 腐蚀性的 HF。因此, 采用更加绿色的途径制备氮掺 杂中空多孔碳球氧化物模拟酶值得进一步探索。

本研究以价格低廉且易去除的碳酸钲微球作为 模板剂, 多巴胺作为氮源与碳源, $\mathrm{KOH}$ 为活化剂, 通过稀盐酸 $(\mathrm{HCl})$ 去模板, 制备出了氮掺杂中空 碳球 (N-HCSs) 氧化物模拟酶。以 $\mathrm{TMB}$ 为底物, 研究了其催化活性及机制。

\section{1 材料与方法}

\section{1 实验试剂}

碳酸钙微球 $\left(\mathrm{CaCO}_{3}\right)$, 广西碳酸钻产业化工程 院有限公司自制; 多巴胺 (PDA), 分析纯, 购自美国 Sigma 试剂公司; 3,3',5,5'-四甲基联苯胺（TMB）, 生物专用, 购自上海润捷化学试剂有限公司; 氢氧 化钾 $(\mathrm{KOH})$ 、盐酸 $(\mathrm{HCl})$ 、乙酸 $(\mathrm{HAc})$ 、乙酸钠 $(\mathrm{NaAC})$, 均为分析纯, 购自广东光华科技股份有 限公司。

\section{2 氮掺杂中空碳球（N-HCSs）的制备}

取 $0.1 \mathrm{~g}$ 碳酸钙微球和 $1.0 \mathrm{~g}$ 盐酸多巴胺盐分别 溶于 $500 \mathrm{~mL}$ 去离了水中低转速搅拌 $10 \mathrm{~min}$, 将两 者混合加入 $1 \mathrm{~mL}$ 氨水与 $10 \mathrm{~mL}$ 乙醇, 在室温下低 速摚拌 $5 \mathrm{~h}$, 获得 $\mathrm{CaCO}_{3} @ \mathrm{PDA}$ 微球, 将其置于管式 炉内在氮气氛围中, 以 $5{ }^{\circ} \mathrm{C} / \mathrm{min}$ 的速度从室温升至 $750{ }^{\circ} \mathrm{C}$, 恒温 $1 \mathrm{~h}$, 自然冷却后用稀盐酸去除模板, 制备氮掺杂中空多孔碳球 (N-HCS); 为了提高 N-HCS 的比表面积和孔容, 将适量的氢氧化钾 $(\mathrm{KOH})$ 与 $\mathrm{CaCO}_{3} @ \mathrm{PDA}$ 微球混匀后置于管式炉中 进行碳化, 用稀盐酸去除模板后得到氮掺杂中空多 孔碳球 (N-HCS-1); N-HCS 和 N-HCS-1 两种材料 统称为 $\mathrm{N}-\mathrm{HCSS}$ 。

\subsection{N-HCSs 过氧化物模拟酶活性的研究}

将 $1 \mathrm{~mL}$ 的 HAC-NaAC 缓冲溶液 $(\mathrm{pH}=4,0.01$ $\mathrm{mol} / \mathrm{L}), 20 \mu \mathrm{L}$ TMB 乙醇溶液 $(0.042 \mathrm{mmol} / \mathrm{L})$, 一 定量的 N-HCSs, 分别依次加入到 $2 \mathrm{~mL}$ 的小试管中, 在 $30{ }^{\circ} \mathrm{C}$ 反应 $10 \mathrm{~min}$, 过滤, 用 UV-Vis 分光光度计, 在波长 $652 \mathrm{~nm}$ 下测定滤液的吸光值。分别考察材料 浓度 $(0.1 \sim 1 \mathrm{mg} / \mathrm{mL}) 、 \mathrm{pH}$ 值 $(2 \sim 6)$ 、温度 $\left(20{ }^{\circ} \mathrm{C}\right.$ $\sim 70{ }^{\circ} \mathrm{C}$ ) 对 N-HCS 过氧化物模拟酶催化活性的影 响。

\section{4 稳态动力学及其机理研究}

在最佳条件下, 对 N-HCSs 氧化物模拟酶分别 进行动力学研究 ${ }^{[25]}$, 采用 Lineweaver-Burk 作图分别 求得 N-HCSs 过氧化物模拟酶的 $K_{\mathrm{m}}$ 和 $V_{\mathrm{m}}$ 。

在体系中通入氮气, 加入异丙醇 (IPA)、过氧 化氢酶 $(\mathrm{CAT})$ 、超氧化物歧化酶 (SOD) 分别作为 $\mathrm{OH} 、 \mathrm{H}_{2} \mathrm{O}_{2}$ 和 $\mathrm{O}_{2} \cdot$-的清除剂, 探究 N-HCSs 氧化物 模拟酶催化反应机理 ${ }^{[26]}$ 。

\section{5 分析表征}

采用日立 5-3400 型的扫描电子显微镜(SEM) 和 FEITECNAI-G2-F30 的场发射透射电子显微镜 
(TEM)作形貌分析。使用 Brunauer-Emmett-Teller (BET)方法, 在 $77 \mathrm{~K}$ 下通过 Micromeritics-ASA2420 氮气吸附脱附仪测定 $\mathrm{N}_{2}$ 吸附-脱附曲线后, 计算 N-HCSs 比表面积。采用 ESCALAB 250XI+X 射线 光电子能谱仪进行元素组成及化学态分析, $X$ 射线 源为单色化 $\mathrm{Al} \mathrm{K \alpha}$ 线源, 测试真空度为 $10^{-8} \mathrm{a}$ 。采用 Burker A300 电子顺磁共振波谱仪 (ESR), 以 BMPO

( 5-tert-Butoxycarbonyl-5-methyl-1-pyrroline-N-oxid $\mathrm{e}, \mathrm{C}_{10} \mathrm{H}_{17} \mathrm{NO}_{3}$ ) 为自由基捕获剂, 测定氧化物模拟 酶催化反应中生成的自由基。

\section{2 结果与讨论}

\subsection{N-HCSs 的形貌和结构分析}

图 1（a）为 N-HCSs 氧化物模拟酶的制备及其 催化反应的示意图。如图 1（b）所示, N-HCS 具有 良好的球形结构, 粒径均一, 约为 $510 \mathrm{~nm}$ 。从 N-HCS 的 EDX 元素映射图可以看出, $\mathrm{C} 、 \mathrm{~N} 、 \mathrm{O}$ 三种元素 在整个空间内分布均匀（图 1 (c c e ) 。

(a)
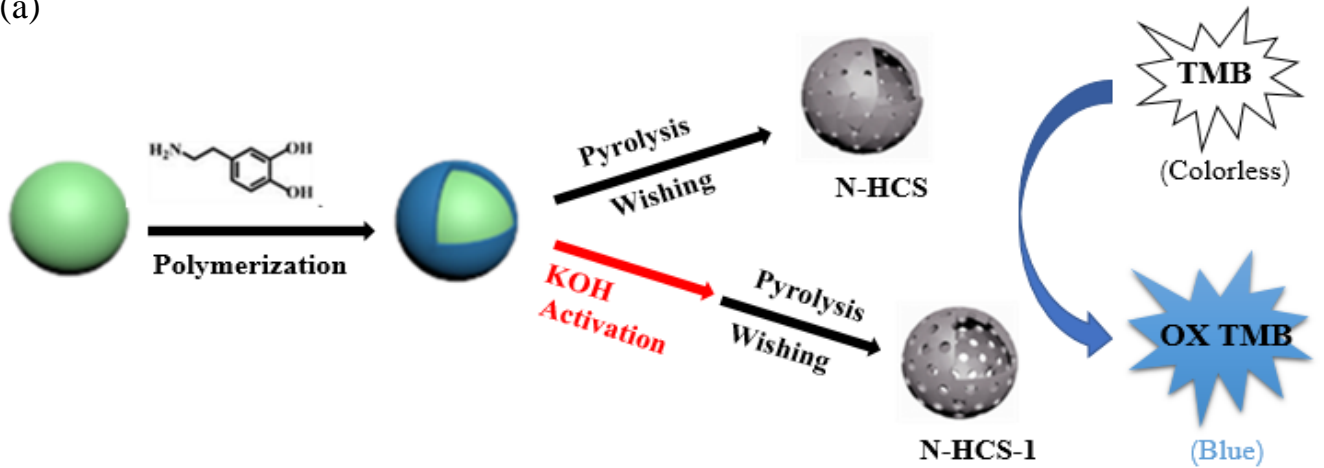

oxidase-like activity
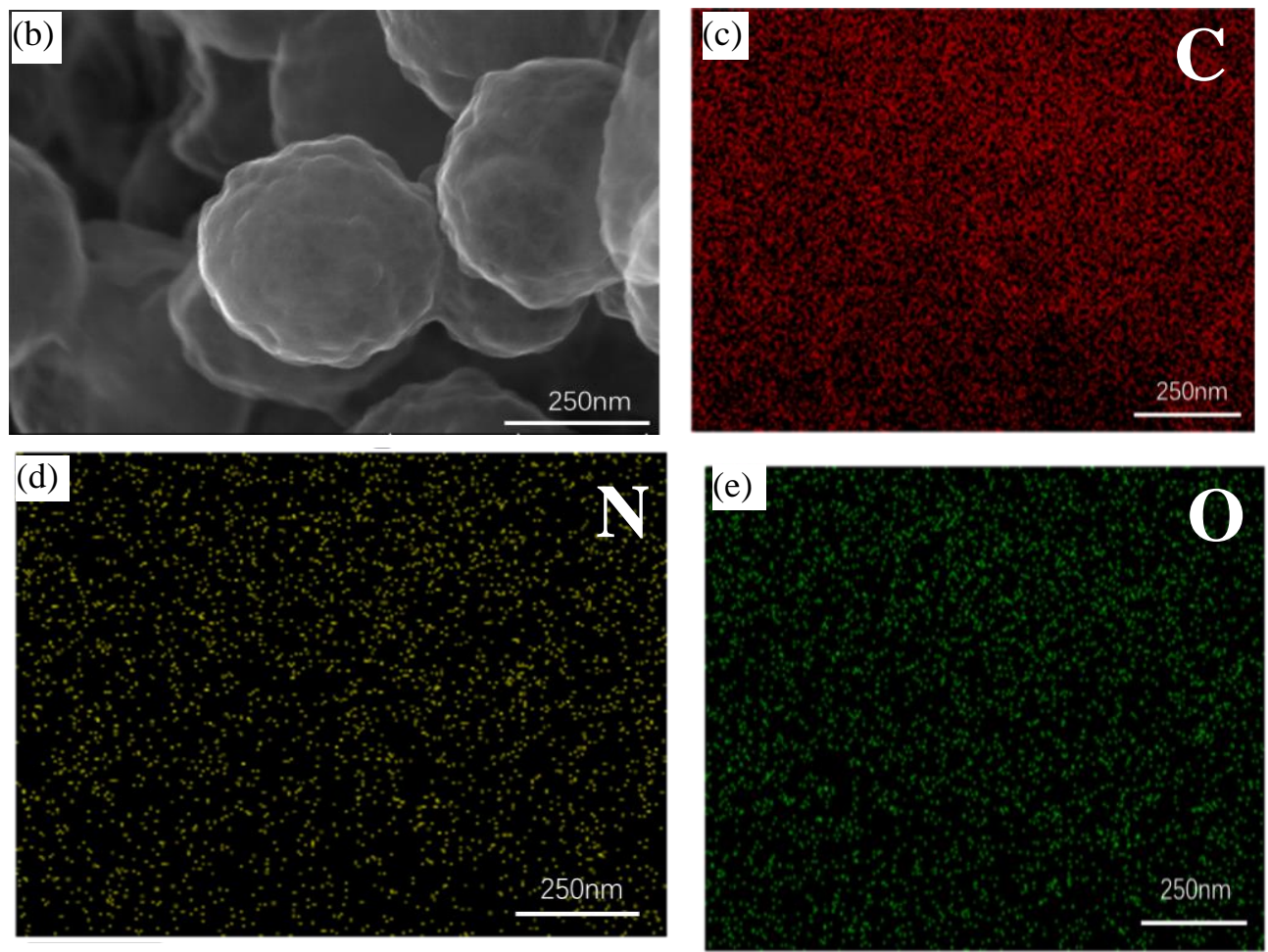

图 $1 \mathrm{~N}-\mathrm{HCSs}$ 的形貌和结构分析

Fig. 1 Morphologies and components of N-HCSs

(a) Schematic presentation for the oxide enzyme mimetic activity of N-HCSs; (b) STEM images of N-HCS;

(c) C, (d) $\mathrm{N}$ and (e) O EDX mappings of N-HCS

通过对比图 2（a）和（b) 可知, 经过 $\mathrm{KOH}$ 活 化后的少部分氮掺杂中空碳球虽然发生轻微的破 
损，但仍然维持球形结构。在碱性条件下，盐酸多 巴胺氧化自聚，形成包覆层，得到具有壳核结构的 $\mathrm{CaCO}_{3} @ \mathrm{PDA}$ 复合微球, 壳层厚度约为 $76 \mathrm{~nm}$ (图 2 (c))。在对 $\mathrm{CaCO}_{3} @ \mathrm{PDA}$ 复合微球高温陆烧过程 中 $\mathrm{CaCO}_{3}$ 分解生成 $\mathrm{CaO}$ 和大量 $\mathrm{CO}_{2}$, 故碳酸钻微球
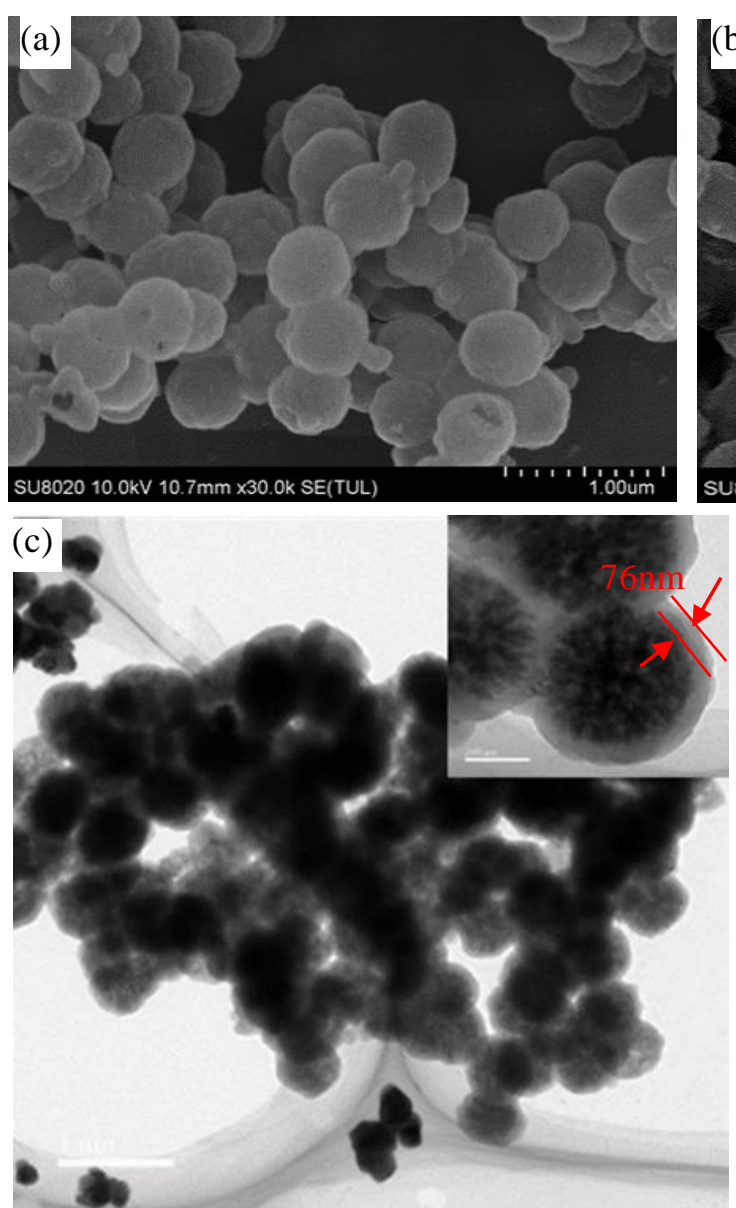

图 2 (a) N-HCS 和 (b) N-HCS-1 的 SEM 照片; (c) $\mathrm{CaCO}_{3} @ \mathrm{PDA}$ 和 (d) N-HCS 的 TEM 照片

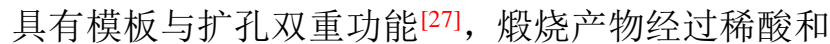
水洗涤去除残余的 $\mathrm{CaO}$ ，得到的中空多孔氮掺杂碳 球（N-HCSs），碳化去模板后壳层发生收缩，壳层 厚度约为 $60 \mathrm{~nm}$, 同时, 也可以观察到 N-HCS 的内 部具有丰富的孔状结构（图 2(d))。

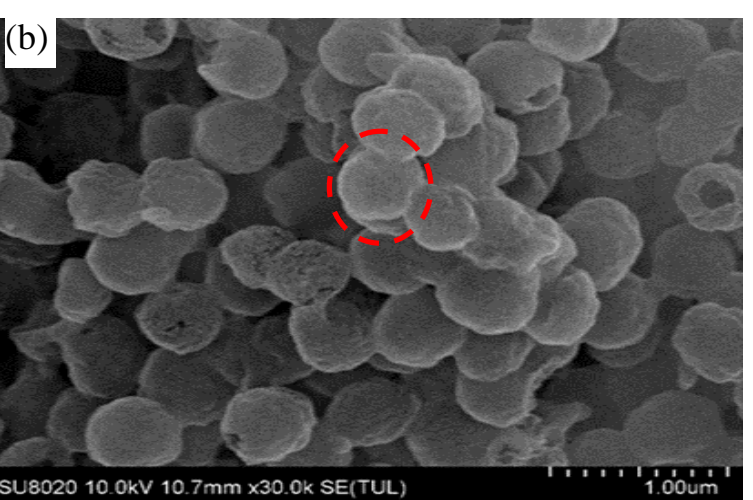

(d)

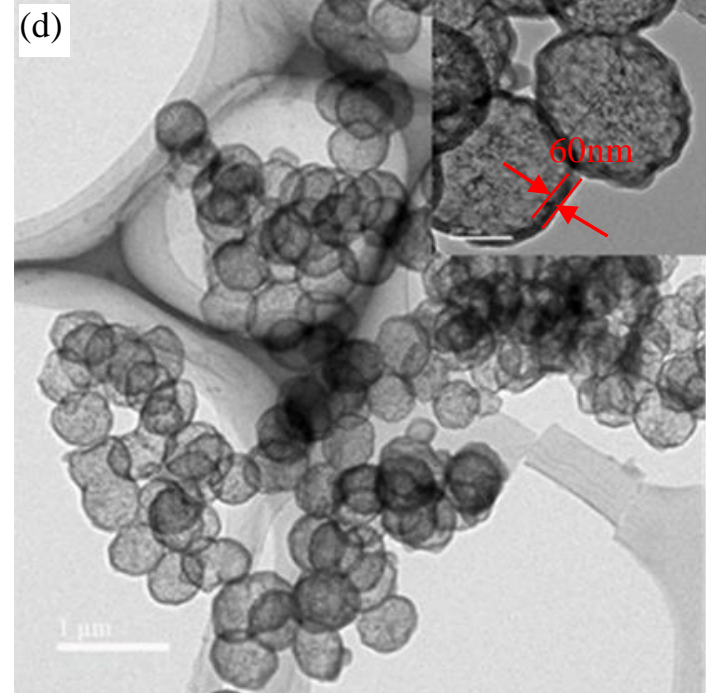

Fig. 2 SEM images of (a) N-HCS and (b) N-HCS-1, and TEM images of (c) $\mathrm{CaCO}_{3} @$ PDA and (d) N-HCS

$\mathrm{N}-\mathrm{HCSs}$ 的 XPS 表征结果如图 3 所示, N-HCSs 主要含有 $\mathrm{C} 、 \mathrm{~N} 、 \mathrm{O}$ 三种元素，与 $\mathrm{N}-\mathrm{HCS}$ 相 比,N-HCS-1 的 C、N 含量增加, $\mathrm{O}$ 含量下降（图 3 (a))，说明 $\mathrm{KOH}$ 活化扩孔的同时加快了材料表面 含氧官能团的脱除 ${ }^{[28]}$ 。从图 3(b,c) 可知, 位于 $\mathrm{N} 1 \mathrm{~s}$ 光谱 398.5、400.1 和 $401.1 \mathrm{eV}$ 的三个峰, 分别 对应于吡啶氮、吡咯氮和石墨氮。 $\mathrm{KOH}$ 活化使得吡 啶氮和吡咯氮含量分别由原来的 $18.73 \%$ 和 $56.54 \%$ 增加到 $20.21 \%$ 和 $61.23 \%$, 而石墨氮含量则由 $24.73 \%$ 下降至 18.56\%（图 3（d））。在氮掺杂碳材
料中，氧还原反应 $(\mathrm{ORR})$ 的活性位点是与吡啶氮和 吡咯氮相邻的具有路易斯碱度的碳原子，则吡啶氮 和吡咯氮的含量相应增加, 可为 ORR 反应提供更多 活性位点 ${ }^{[29]}$, 从而促进 $\mathrm{O}_{2}$ 分子的吸附, 加速活性 氧 (ROS) 的生成, 提高其催化氧化能力。 

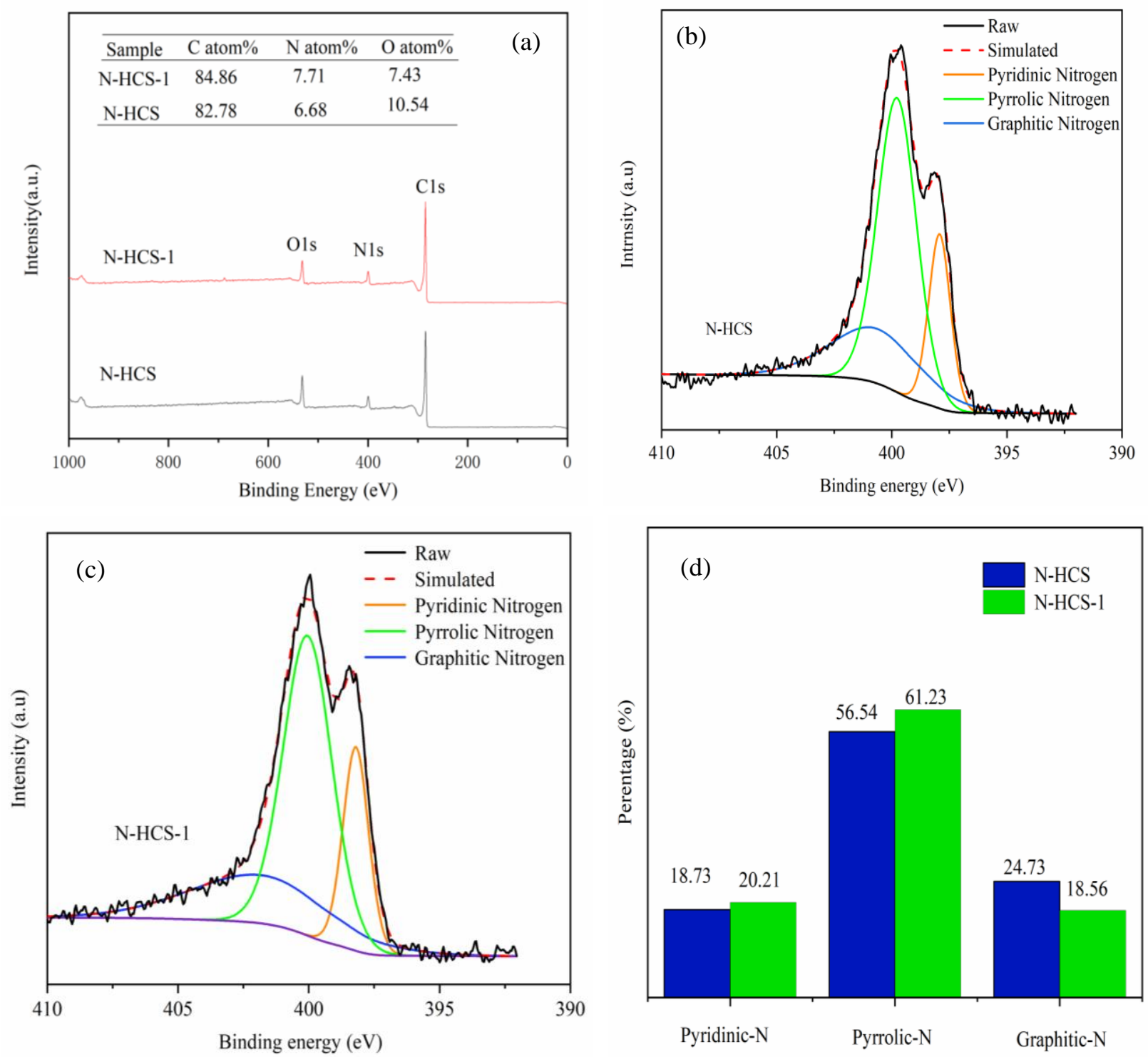

图 3 (a) N-HCS 和 N-HCS-1 的 XPS 全谱, (b) N-HCS 和(c) N-HCS-1 的 N1s 谱, (d) N-HCS 和 N-HCS-1 的 N 元素组成

Fig.3 (a) XPS full spectra of N-HCS and N-HCS-1; N1s spectra of (b) N-HCS and (c) N-HCS-1; (d) N species contents of N-HCS and N-HCS-1

图 4（a）和（b）是 N-HCSs 的 $\mathrm{N}_{2}$ 吸附脱附等 温曲线和 HK 模型孔径分布图。由图 4（a）可知, N-HCS 和 N-HCS-1 表现出 IV 型等温线的特征, 表 明两者都具有分级多孔结构。根据图 4（b）样品的 $\mathrm{HK}$ 模型孔径分布图可知, 经 $\mathrm{KOH}$ 活化后的
N-HCS-1 微孔含量下降, 介孔含量增加, 可减小底 物与催化剂之间的传质阻力。由表 1 数据可知, N-HCS-1 的比表面积和孔容均高于 N-HCS, 故 N-HCS-1 能提供更多的催化反应活性位点。 

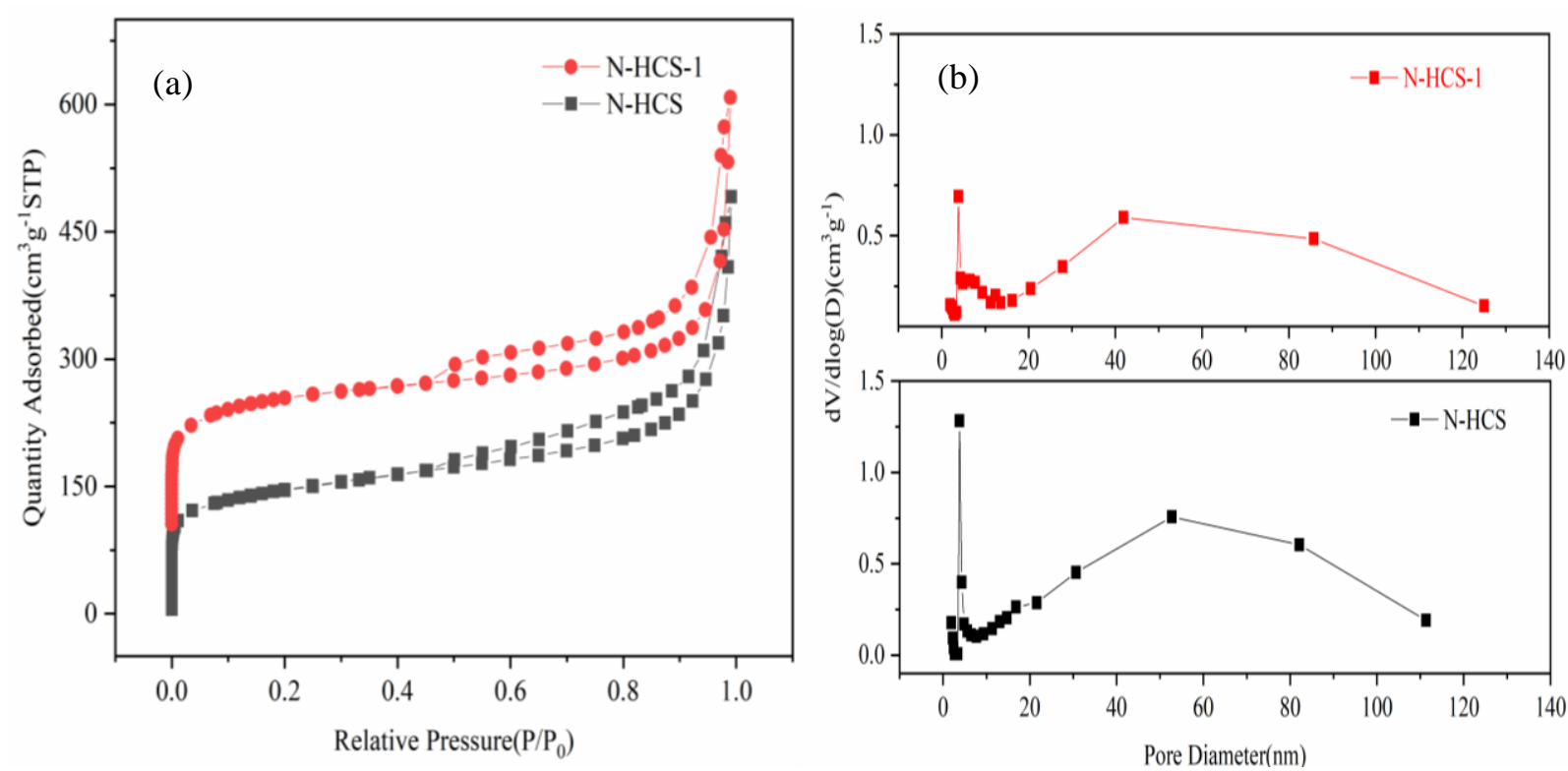

图 4 (a) N-HCS 和 N-HCS-1 的 $\mathrm{N}_{2}$ 吸附脱附等温曲线及（b） HK 模型孔径分布图

Fig. 4 (a) Nitrogen absorption-desorption isotherm curves of N-HCS and N-HCS-1, (b) HK model corresponding pore size distribution curves of N-HCS and N-HCS-1

表 1 N-HCS 和 N-HCS-1 材料的孔结构参数

Table 1 Pore structure parameters of N-HCS and N-HCS-1

\begin{tabular}{ccccccc} 
Sample & \multicolumn{2}{c}{ Specfic suface $/\left(\mathrm{m}^{2} \cdot \mathrm{g}^{-1}\right)$} & $V_{\mathrm{t}} /\left(\mathrm{cm}^{3} \cdot \mathrm{g}^{-1}\right)$ & $\left.V_{\mathrm{m}} / \mathrm{cm}^{3} \cdot \mathrm{g}^{-1}\right)$ & $S_{\mathrm{m}} /\left(\mathrm{m}^{2} \cdot \mathrm{g}^{-1}\right)$ & $\left(\mathrm{S}_{\mathrm{m}} / \mathrm{S}_{\mathrm{t}}\right) / \%$ \\
\cline { 2 - 3 } & Langmuir & BET & & & & \\
\hline N-HCS & 685.6 & 490.1 & 0.651 & 0.109 & 230.51 & 45.49 \\
N-HCS-1 & 730.0 & 518.4 & 0.732 & 0.111 & 228.16 & 44.01 \\
\hline
\end{tabular}

\subsection{N-HCSs 氧化物模拟酶活性的探究}

选择 3,3',5,5'-四甲基联苯胺(TMB)作为显色底 物, N-HCSs 作为催化剂, 探究其氧化物模拟酶的 催化特性。如图 5(a)所示, TMB 单独在 NaAC-HAC 缓冲溶液中，不能发生显色反应，而 N-HCSs 能够 催化 TMB 氧化成蓝色的产物 ox-TMB，在 $652 \mathrm{~nm}$ 处有一个特征吸收峰, 说明 N-HCSs 具有氧化物模 拟酶活性，且通过 $\mathrm{KOH}$ 活化扩孔有效提高了氮掺 杂中空碳球氧化物模拟酶的催化活性。

氧化物模拟酶的催化活性与其浓度、 $\mathrm{pH}$ 、温度 均有关。由图 5(b) 可知, 随着 N-HCSs 的浓度增加, 其类氧化物酶催化活性不断增加, 当 N-HCSs 大于
$0.8 \mathrm{mg} / \mathrm{mL}$ 时, 催化活性呈下降趋势, 这可能是由 于催化剂浓度过高时, 容易团聚, 导致底物与催化 剂之间的传质阻力增加, 不利于催化反应进行 ${ }^{[30]}$ 。 $\mathrm{pH}$ 为 3 时 N-HCSs 氧化物模拟酶的催化活性最高 （图 5 (c))，相比贵金属基的氧化物模拟酶（最适 $\mathrm{pH}$ 为 4.5 ), 其催化反应的最适 $\mathrm{pH}$ 更偏酸性。在反 应温度为 $20 \sim 70{ }^{\circ} \mathrm{C}$ 较大的温度范围内, N-HCSs 氧 化物模拟酶仍然可以保留 $90 \%$ 的催化活性(图 5

(d)), 说明 N-HCSs 氧化物模拟酶对温度的适应 性明显优于天然氧化物酶。此外, N-HCSs 氧化物 模拟酶具有良好的稳定性, 可多次循环使用 (图 6), 故其在实际应用中具有较大的潜力。 

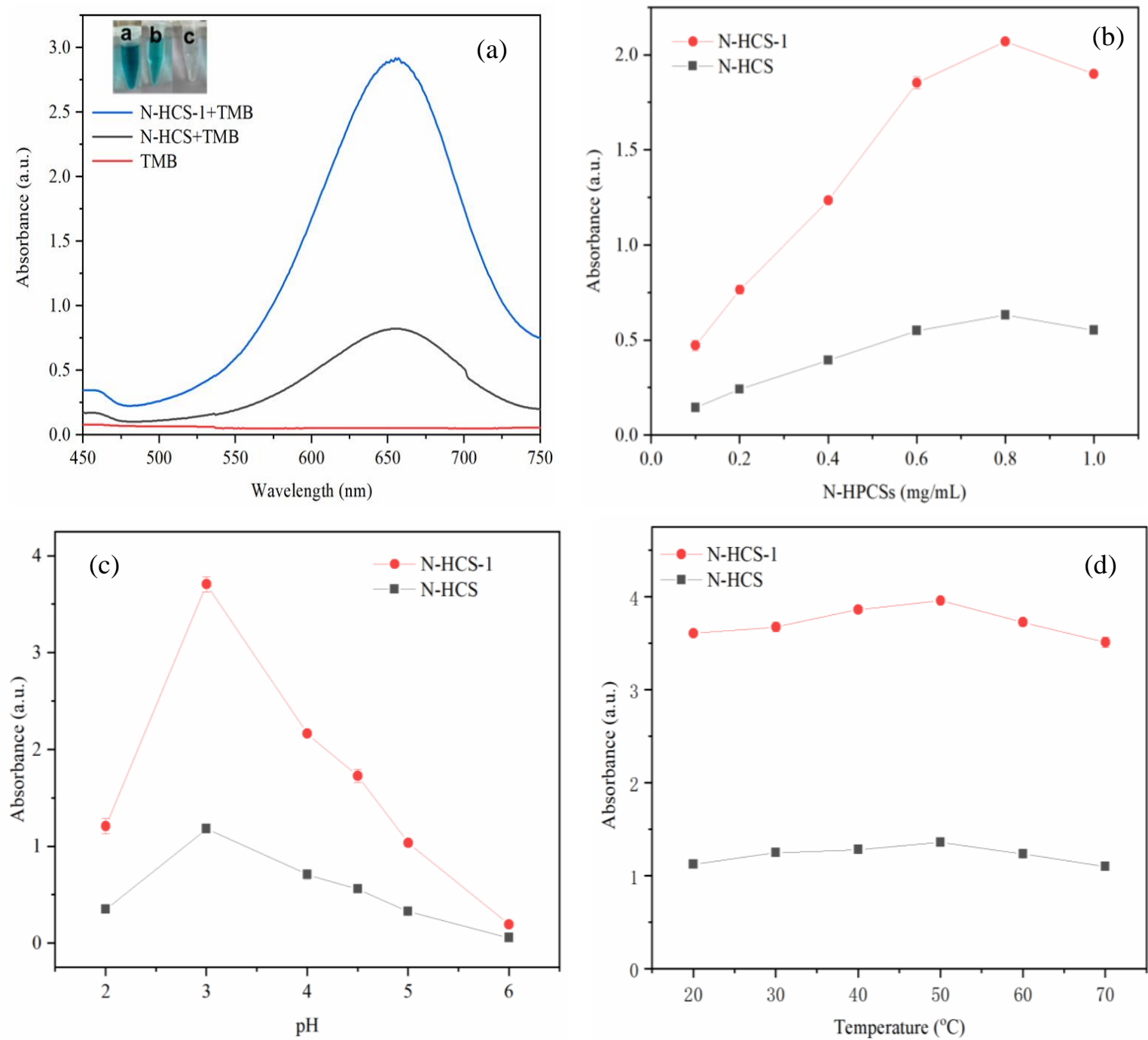

图 5 (a)不同体系的紫外可见吸收光谱，(b)催化剂浓度、（c） pH、（d）温度对 N-HCSs 氧化物模拟酶活性的影响

Fig. 5 (a) UV-Vis absorption spectra of different systems, and effects of (b) catalyst concentration, (c) pH and (d) temperature on the oxidase-like activity of N-HCSs

\section{3 稳态动力学和催化机理研究}

通过改变体系中 TMB 浓度对其稳态动力进行 研究, 由图 (6) 可知, N-HCSs 氧化物模拟酶催化 反应遵循典型的 Michaelis-Menten 动力学方程, 通 过 Lineweaver-Burk 作图后分别计算出 N-HPCS 和 N-HPCS-1 的米氏常数 $\left(K_{\mathrm{m}}\right)$ 和最大反应速率 $\left(V_{\mathrm{m}}\right)$, 结果列于表 3。N-HCS-1 的米氏常数小于 N-HCS, 表明 $\mathrm{KOH}$ 活化可增强材料对底物 TMB 的亲和能 力。相比文献所报道的氮掺杂碳球 ${ }^{[4]}$, 中空多孔氮 掺杂碳球对底物 TMB 的亲和能力显著增强, 这可 归因于其中空的多级多孔结构。

$\mathrm{N}-\mathrm{HCSs}$ 氧化物模拟酶的催化特性从本质上应 该与体系中的 $\mathrm{O}_{2}$ 及生成的活性氧 (ROS) 有关 ${ }^{[31]}$ 。 在体系中通入 $\mathrm{N}_{2}$, 如图 7 (a) 所示, 当 N-HCSs
处于 $\mathrm{N}_{2}$ 环境中时, 类氧化物酶活性因缺氧而被抑 制, 这说明 $\mathrm{O}_{2}$ 在 N-HCSs 氧化物模拟酶催化反应中 起重要作用, 且对 N-HCS-1 的催化活性抑制效果更 明显, 这验证了 N-HCS-1 能为 $\mathrm{O}_{2}$ 吸附提供更多的 吸附位点的推测。在体系引入异丙醇和 CAT 后对催 化反应的影响不大 (图 7 (b c ) ), 而在加入 SOD 后, N-HCSs 氧化物模拟酶活性明显降低(图 7(d)), 表明 $\mathrm{O}_{2} \cdot$ - 是 N-HCSs 氧化物模拟酶催化反应中主要 活性氧基团，与已报道的贵金属基、金属氧化物基 氧化物模拟酶的催化机理相一致 ${ }^{[32-33]}$ 。 


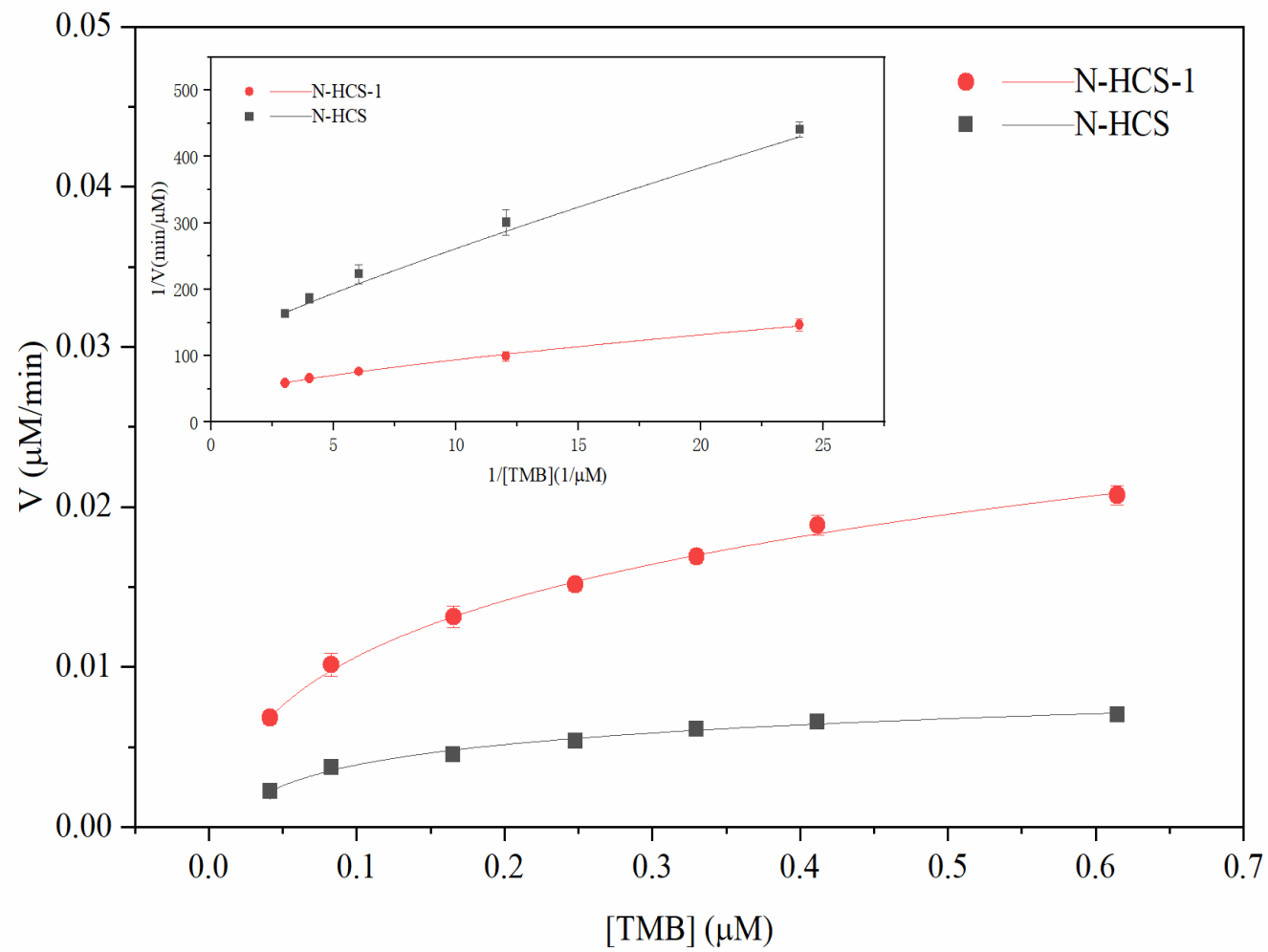

图 6 N-HCS 和 N-HCS-1 稳态动力学分析

Fig.6 Steady-state kinetic assay of N-HCS and N-HCS-1

表 $2 \mathrm{~N}-\mathrm{HCSs}$ 过氧化物酶动力学参数

Table 2 Pore structure parameters of N-HCSs

\begin{tabular}{ccccc}
\hline Catalyst & Substrate & $K_{\mathrm{m}} /\left(\mathrm{mmol} \cdot \mathrm{L}^{-1}\right)$ & $V_{\mathrm{m}} /\left(\times 10^{-8} \mathrm{~mol} \cdot \mathrm{L}^{-1} \cdot \mathrm{S}^{-1}\right)$ & Ref. \\
\hline N-PCS & TMB & 0.095 & 5.20 & {$[4]$} \\
His@ AuNCs & TMB & 0.041 & 6.21 & {$[34]$} \\
Pt Au DNPs & TMB & 0.22 & 2.82 & {$[35]$} \\
Acr- Mes & TMB & 0.129 & 2.68 & {$[36]$} \\
N-HCS & TMB & 0.1049 & 4.69 & This work \\
N-HCS-1 & TMB & 0.0825 & 5.98 & This work \\
\hline
\end{tabular}



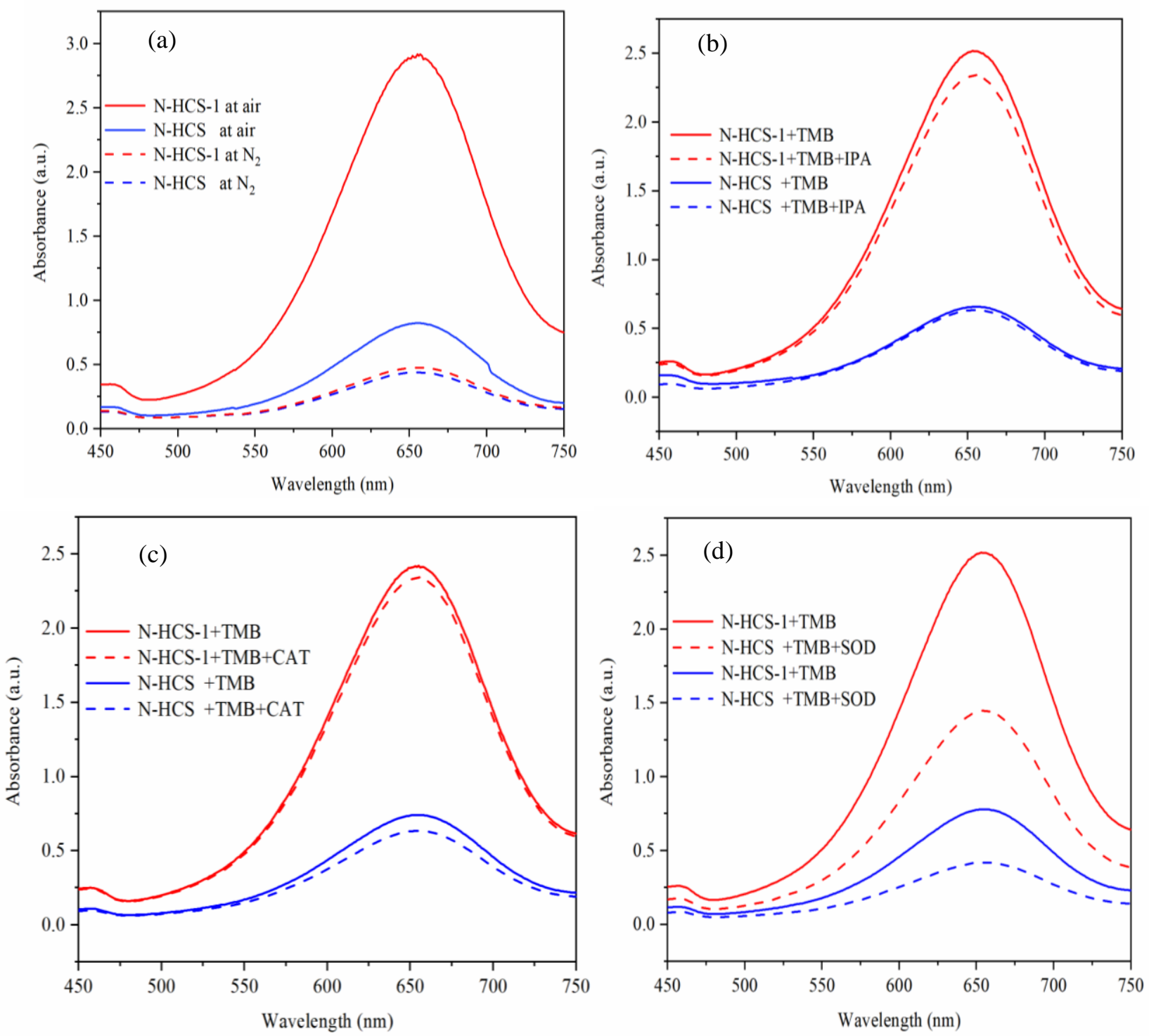

图 7 在体系中 (a) 通入氮气后的紫外光图谱, 及加入不同活性氧清除剂 (b)异丙醇(IPA)、(c)过氧化氢酶 (CAT)、(d) 超 氧化物歧化酶（SOD）对 N-HCS 和 N-HCS-1 类氧化物酶活性的影响

Fig.7 (a) Absorption spectra of the solution containing TMB and N-HCS and N-HCS-1 under $\mathrm{N}_{2}$-saturated conditions, and effect of scavengers (b) IPA, (c) CAT and (d) SOD on the catalytic oxidation of TMB by the N-HCS and N-HCS-1

为了进一步鉴定 N-HCSs 氧化物模拟酶的催化 活性是否与生成 $\mathrm{O}_{2}{ }^{-}$相关, 我们采用电子自旋共振 光谱 (ESR), 以 BMPO (5-tert-Butoxycarbonyl-5methyl-1-pyrroline- $\mathrm{N}$-oxide, $\mathrm{C}_{10} \mathrm{H}_{17} \mathrm{NO}_{3}$ ）作为自旋 阱来探测 $\mathrm{O}_{2}{ }^{-}$的生成。如图 8(a)所示, N-HCS 和 $\mathrm{N}-\mathrm{HCS}-1$ 在 $\mathrm{pH}$ 为 3 的 HAC-NaAC 缓冲溶液中放置 $10 \mathrm{~min}$ 后的 $\mathrm{ERS}$ 图谱均显示了 $\mathrm{BMPO}-\mathrm{O}_{2}{ }^{-}$加合物的 六重特征峰, 说明两者体系中生成了 $\mathrm{O}_{2}{ }^{\circ}$ 。可以观
察到 N-HCS-1 的特征峰明显高于 N-HCS, 这说明 N-HCS-1 催化生成 $\mathrm{O}_{2}{ }^{-}$的能力较强。

综上, N-HCSs 氧化物模拟酶的催化反应机制 可以解释为: N-HCSs 将溶解的 $\mathrm{O}_{2}$ 吸附到表面, 催 化生成 $\mathrm{O}_{2}{ }^{-}, \mathrm{O}_{2}{ }^{-}$氧化底物 TMB, 生成蓝色 oxTMB 的产物（图 8（b)）。N-HCS-1 具有更高的类氧化物 酶活性可归因于其能提供更多的反应活性位点, 可 促进 $\mathrm{O}_{2}$ 的吸附, 加速 $\mathrm{O}_{2}^{-}$的生成。 


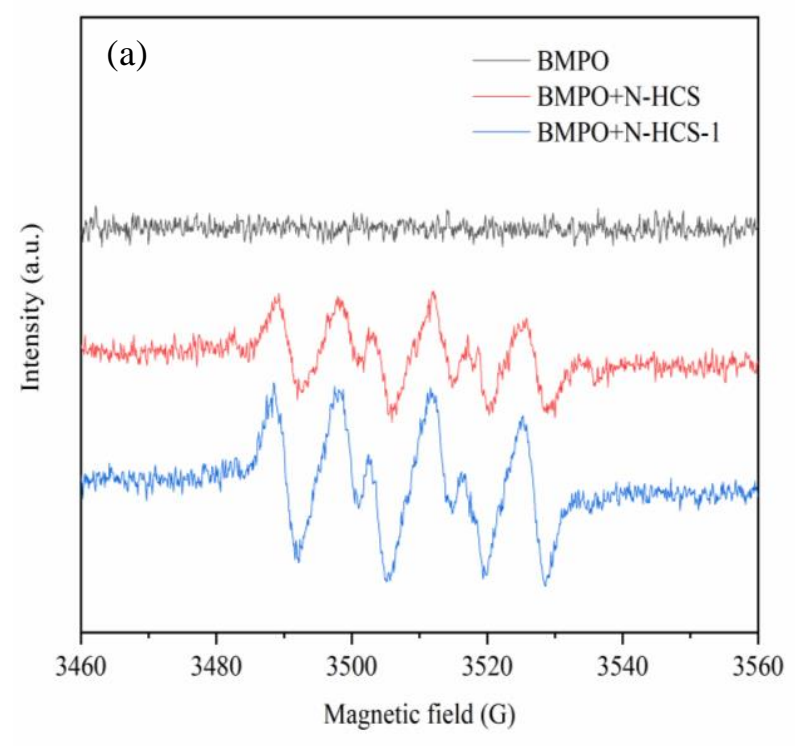

图 8（a） N-HCS 和 N-HCS-1 的电子顺磁共振图谱（ESR）及（b） N-HCS 氧化物模拟催化机理图 (b)

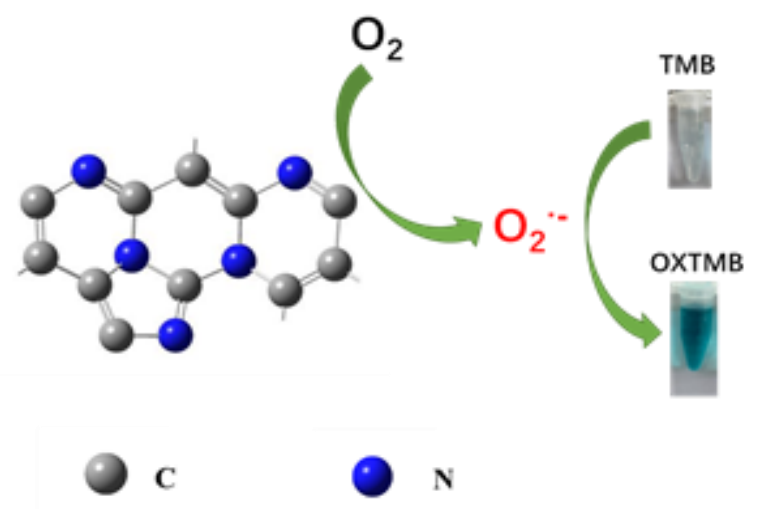

dase-like activity of the N-HCS and N-HCS-1

\section{3 结论}

本研究采用硬模板法制备出氮掺杂中空碳球 （N-HCSs）氧化物模拟酶，对其类酶催化活性及其 催化机理作了研究。结果表明, 经过 $\mathrm{KOH}$ 活化的 N-HCS-1 具有高含氮量、高比表面积和大孔容等特 性，因此其类氧化物酶催化活性较 N-HCS 的催化活 性更强。N-HCSs 氧化物模拟酶的最佳催化条件为催 化剂浓度 $0.8 \mathrm{mg} / \mathrm{L} 、 \mathrm{pH} 3.0 、 50{ }^{\circ} \mathrm{C}$ 。 N-HCSs 类氧化 物酶催化氧化反应中，超氧阴离子 $\left(\mathrm{O}_{2}{ }^{-}\right)$是主要的 活性氧基团。由于 N-HCSs 氧化物模拟酶催化反应需 要在强酸条件下才能进行, 故如何拓宽该催化反应 的最优酸碱度还有待进一步研究。

\section{参考文献:}

[1] LIN Y, REN J, QU X, et al. Catalytically active nanomaterials: a promising candidate for artificial enzymes. Accounts of Chemical Research, 2014, 47(4): 1097-1105.

[2] GAO L, ZHUANG J, NIE L, et al. Intrinsic peroxidase-like activity of ferromagnetic nanoparticles. Nature Nanotechnology, 2007, 2(9): 577-583.

[3] WEI X, CHEN J, ALI M C, et al. Cadmium cobaltite nanosheets synthesized in basic deep eutectic solvents with oxidase-like, peroxidase-like, and catalase-like activities and application inthe colorimetric assay of glucose. Microchimica Acta, 2020, 187(6): 314-325.

[4] FAN K, XI J, FAN L, et al. In vivo guiding nitrogen-doped carbon nanozyme for tumor catalytic therapy. Nature Communications, 2018, 9: 1440.
[5] CHEN Z, YIN J-J, ZHOU Y-T, et al. Dual enzyme-like activities of iron oxide nanoparticles and their implication for diminishing cytotoxicity. ACS Nano, 2012, 6(5): 4001-4012.

[6] WEI H, WANG E. Nanomaterials with enzyme-like characteristics (nanozymes): next-generation artificial enzymes. Chemical Society Reviews, 2013, 42(14): 6060-6093.

[7] ZHANG X, MAO X, LI S Q, et al. Tuning the oxidase mimics activity of manganese oxides via control of their growth conditions for highly sensitive detection of glutathione. Sensors and Actuators B-chemical, 2018, 258: 80-87.

[8] WANG Y, ZHANG Z, JIA G, et al. Elucidating the mechanism of the structure-dependent enzymatic activity of Fe-N/C oxidase mimic. Chemical Communications, 2019, 55(36): 5271-5274.

[9] HE W, HAN X, JIA H, et al. AuPt alloy nanostructures with tunable composition and enzyme-like activities for colorimetric detection of bisulfide. Scientific Reports, 2017, 7: 40103.

[10] GE C, FANG G, SHEN X, et al. Facet energy versus enzyme-like activities: the unexpected protection of palladium nanocrystals against oxidative damage. ACS Nano, 2016, 10(11): 10436-10445.

[11] HU Y, GAO X J, ZHU Y, et al. Nitrogen-doped carbon nanomaterials as highly active and specific peroxidase mimics. Chemistry of Materials, 2018, 30(18): 6431-6439.

[12] MU J, LI J, ZHAO X, et al. Cobalt-doped graphitic carbon nitride with enhanced peroxidase-like activity for wastewater treatment. RSC Advances, 2016, 6(42): 35568-35576.

[13] AHMWD A, JOHN P, NAWAZ M H, et al. Zinc-doped mesoporous graphitic carbon nitride for the colorimetric detection of hydrogen peroxide. ACS Nano Materials, 2019, 2(8): 5156-5168.

[14] ZHANG H, LIANG X, HAN L, et al. "Non-naked" gold with glu- 
cose oxidase-like activity: a nanozyme for tandem catalysis. Small, 2018, 14(44): e1803256.

[15] LI R, LEI C, ZHAO X-E, et al. A label-free fluorimetric detection of biothiols based on the oxidase-like activity of $\mathrm{Ag}^{+}$ions. Sectrochimica Acta art A: Molecular \& Biomolecular Spectroscopy, 2018, 188: $20-25$.

[16] FENG J Y, HUANG P, WU F-Y, et al. Gold-platinum bimetallic nanocluster with enhanced peroxidase-like activity and its integrated agarose hydrogel-based sensing platform for colorimetric analysis of glucose level in serum. Analyst, 2017, 142(21): 4106-4115.

[17] SHEN X, LIU W, GAO X, et al. Mechanisms of oxidase and superoxide dismutation-like activities of gold, silver, platinum, and palladium, and their alloys: a general way to the activation of molecular oxygen. Journal of the American Chemical Society, 2015 , 137(50): 15882-15891.

[18] HUANG L, ZHANG W, CHEN K, et al. Facet-selective response of trigger molecule to $\mathrm{CeO}_{2}\{110\}$ for up-regulating oxidase-like activity. Chemical Engineering Journal, 2017, 330(17): 746-752.

[19] ZHAO M, TAO Y, HUANG W, et al. Reversible pH switchable oxidase-like activities of $\mathrm{MnO}_{2}$ nanosheets for a visual molecular majority logic gate. Physical Chemistry Chemical, 2018, 20(45): 28644-28648

[20] FAN Y, SHI W, ZHANG X, et al. Mesoporous material-based manipulation of the enzyme-like activity of $\mathrm{CoFe}_{2} \mathrm{O}_{4}$ nanoparticles. Journal of Materials Chemistry A, 2014, 2(8): 2482-2486.

[21] DU J, LIU L, YU Y, et al. Hollow carbon sphere with tunable structure by encapsulation pyrolysis synchronous deposition for cefalexin adsorption. Journal of Inorganic Materials, 2020, 35(5): 608-616.

[22] WANG N, SONG H, REN H, et al. Partly nitrogenized nickel oxide hollow spheres with multiple compositions for remarkable electrochemical performance. Chemical Engineering Journal, 2019, 358: $531-539$.

[23] GONG K, DU F, XIA Z, et al. Nitrogen-doped carbon nanotube arrays with high electrocatalytic activity for oxygen reduction. Science, 2009, 323(5915): 760-764.

[24] ZHANG Y, YANG H, CHI Q, et al. Nitrogen-doped carbon supported nickel nanoparticles: a robust catalyst to bridge the hydrogenation of nitriles and the reductive amination of carbonyl compounds for the synthesis of primary amines. ChemSusChem, 2019, 12(6): $1246-1255$
[25] FAN L, XU X, ZHU C, et al. Tumor catalytic-photothermal therapy with yolk-shell Gold@Carbon nanozymes. ACS Applied Materials \& Interfaces, 2018, 10(5): 4502-4511.

[26] LIANG H-W, ZHUANG X, SEBASTIAN B, et al. Hierarchically porous carbons with optimized nitrogen doping as highly active electro-catalysts for oxygen reduction. Nature Communications, 2014, 5: 4973

[27] HE X, LIU P, LIU J, et al. Facile synthesis of hierarchical N-doped hollow porous carbon whiskers with ultrahigh surface area via synergistic inner-outer activation for casein hydrolysate adsorption. Journal of Materials Chemistry B, 2017, 5: 9211-9218.

[28] MISTAR E M, ALFATAH T, SUPARDAN M D. Synthesis and characterization of activated carbon from Bambusa vulgaris striata using two-step $\mathrm{KOH}$ activation. Journal of Materials Research and Technology, 2020, 9(3): 6278-6286.

[29] WU T, MA Z, LI P, et al. Bifunctional colorimetric biosensors via regulation of the dual nanoenzyme activity of carbonized FeCo-ZIF. Sensors \& Actuators B Chemical, 2019, 290: 357-363.

[30] WANG H, LI S, SI Y, et al. Recyclable enzyme mimic of cubic $\mathrm{Fe}_{3} \mathrm{O}_{4}$ nanoparticles loaded on graphene oxide-dispersed carbon nanotubes with enhanced peroxidase-like catalysis and electrocatalysis. Journal of Materials Chemistry B, 2016, 2: 4442-4448.

[31] WANG Y, ZHANG Z, JIA G, et al. Elucidating the mechanism of the structure-dependent enzymatic activity of $\mathrm{Fe}-\mathrm{N} / \mathrm{C}$ oxidase mimics. Chemical Communications, 2019, 55: 5271-5274.

[32] CHEN M, WANG Z, SHU J, et al. Mimicking a natural enzyme system: cytochrome c oxidase-like activity of $\mathrm{Cu}_{2} \mathrm{O}$ nanoparticles by receiving flectrons from cytochrome. Inorganic Chemistry, 2017, 56(16): 9400-9403.

[33] LIU J, HU X, HOU S, et al. Au@Pt core/shell nanorods with peroxidase and ascorbate oxidase-like activities for improved detection of glucose. Sensors \& Actuators B Chemical, 2012, 166/167: 708-714.

[34] LIU L, DU J, LIU W-E, et al. Enhanced His@ AuNCs oxidase-like activity by reduced graphene oxide and its application for colorimetric and electrochemical detection of nitrite. Analytical \& Bioanalytical Chemistry, 2019, 411(10): 2189-2200.

[35] CHENG Q, YANG Y, YANG L-L, et al. Pt-Au dendritic nanoparticles with high oxidase-like activity for detection of ascorbic acid. Journal of Inorganic Materials, 2020, in press. (DOI: 10.15541/jim2020005)

[36] DU J, WANG J, HUANG W, et al. Visible light-activatable oxidase mimic of 9-mesityl-10-methylacridinium ion for colorimetric detection of biothiols and logic operations. Analytical Chemistry, 2018, 90 (16): 9959-9965. 